\title{
Comunicação
}

\section{Biomassa microbiana e matéria orgânica em solo de Caatinga, cultivado com melão na Chapada do Apodi, Ceará}

\author{
Humbelina Silva Siqueira Lopes ${ }^{1}$, Monalisa Gurgel de Medeiros ${ }^{1}$, Joyce Reis Silva ${ }^{2}$ \\ Francisco Assis Medeiros Júnior ${ }^{1}$, Márcio Neres dos Santos ${ }^{3}$, Rafael Oliveira Batista ${ }^{4}$
}

\section{RESUMO}

O uso contínuo, ao longo dos anos, do sistema convencional de preparo do solo pode alterar os atributos microbiológicos do solo, causando a redução da biomassa e da atividade microbiana. Diante do exposto, o objetivo deste trabalho foi avaliar, sob cultivo do melão (Cucumis melo L.), as alterações microbiológicas do solo, assim como as do teor de matéria orgânica, nas profundidades de 0 a 0,10, 0,10 a 0,20, 0,20 a 0,30 e 0,30 a 0,40 m, ao longo dos anos sob cultivo do melão, durante três, cinco e dez anos, em Cambissolo Háplico eutrófico, a fim de compará-los com os do solo da mata natural (Caatinga). As amostras foram coletadas, aleatoriamente, na Fazenda Melão Doçura, localizada no município de Quixeré, Ceará, e encaminhadas para o Laboratório de Análise de Solos, Água e Planta da UFERSA, Mossoró, Rio Grande do Norte. Determinaram-se os teores de matéria orgânica (MO), o de carbono da biomassa microbiana (CBM) e o quociente microbiano ( $q \mathrm{MIC})$. O delineamento experimental foi o inteiramente casualizado (DIC), disposto em esquema fatorial 4 x 4 (Fator I, anos de cultivo; Fator II, causa de variação na profundidade), com três repetições. Os resultados mostraram que a matéria orgânica aumentou a partir dos cinco anos de cultivo do melão. $\mathrm{O}$ carbono da biomassa microbiana foi elevado nas camadas superficiais, independentemente do tempo de cultivo, por causa da maior disponibilidade da matéria orgânica, água e nutrientes, e o quociente microbiano diminuiu com o tempo, apresentando menor concentração no cultivo de dez anos do melão, discriminando as possíveis alterações decorrentes do uso agrícola do solo.

Palavras-chave: carbono microbiano, manejo do solo, carbono orgânico.

\section{ABSTRACT}

\section{Microbial biomass and organic matter in soil of Caatinga, cultivated with melon in Chapada do Apodi, Ceará State}

The intensive use of the conventional system over the years of soil preparation may change the soil microbial attributes, causing a reduction of biomass and microbial activity. As so, the present study aimed to analyze, under melon (Cucumis melo L.) cultivation, the microbiological changes as well as soil organic matter content in the depth of $0-0.10,0.10-0.20,0.20-0.30$ and $0.30-0.40$ m over the years under melon cultivation during 3,5 and 10 years in eutrophic Cambisol, in order to compare them with the natural forest (Caatinga). The samples were randomly collected at Fazenda Melão Doçura, located in the city of Quixeré, Ceará State and forwarded to the Laboratory of Analysis of Soil, Water and Plant of the UFERSA, Mossoró, Rio Grande do Norte State. The content of organic matter (MO), microbial biomass

\footnotetext{
Recebido para publicação em 20/01/2011 e aprovado em 16/08/2012.

${ }^{1}$ Engenheiros-Agrônomos. Mestrandos da Universidade Federal Rural do Semi-Árido, Avenida Francisco Mota, 572, Bairro Costa e Silva, 59625-900, Mossoró, Rio Grande do Norte, Brasil. humbelinas@hotmail.com; monalisa_gurgel@hotmail.com; juniortib@msn.com

${ }^{2}$ Tecnóloga em fruticultura. Mestranda da Universidade Federal Rural do Semi-Árido, Avenida Francisco Mota, 572, Bairro Costa e Silva, 59625-900, Mossoró, Rio Grande do Norte, Brasil. joytareis@hotmail.com

${ }^{3}$ Engenheiro-Agrônomo, Doutor. Departamento de Solos, Universidade Federal Rural do Semi-Árido, Avenida Francisco Mota, 572, Bairro Costa e Silva, 59625-900, Mossoró, Rio Grande do Norte, Brasil. marcioneres@gmail.com

${ }^{4}$ Engenheiro Agrí́cola, Doutor. Departamento de Ciências Ambientais e Tecnológicas, Universidade Federal Rural do Semi-Árido, Avenida Francisco Mota, 572, Bairro Costa e Silva, 59625-900, Mossoró, Rio Grande do Norte, Brasil. rafaelbatista@ufersa.edu.br (autor para correspondência).
} 
carbon $(\mathrm{CBM})$ and microbial quotient $(q \mathrm{MIC})$ were determined. The experiment was mounted on completely randomized design (DIC), arranged in a factorial scheme 4 x 4 (years of cultivation Factor I, Factor II cause of variation in depth) with three replications. The results showed that organic matter increased from five years of cultivation of the melon. The microbial biomass carbon was higher in the superficial layers independent of the culture period due increased availability of organic matter, water and nutrients and microbial quotient decreased with time, with lower concentration in the 10 years cultivation of the melon, detailing the possible changes arising from agricultural soil use.

Key words: microbial carbon, soil management, organic carbon.

\section{INTRODUÇÃO}

O melão (Cucumis melo L.) é o fruto de uma olerícola de grande importância econômica no Brasil, em especial na região nordeste. Uma das principais vantagens do cultivo no semiárido são as condições edafoclimáticas, nas quais temperatura, luminosidade, umidade relativa e a pequena ocorrência de chuvas favorecem a baixa incidência de doenças e a melhor qualidade dos frutos, tornando, assim, os pólos Assu-Mossoró (RN) e baixo Jaguaribe (CE) os maiores produtores do Brasil. Sua principal área de produção dá-se na Chapada do Apodi, coberta por formações montanhosas e localizada na divisa dos Estados do Rio Grande do Norte e Ceará. A chapada funciona como divisor de águas entre as bacias hidrográficas dos rios Apodi e Jaguaribe, caracterizada por predomínio de Cambissolos derivados de rochas calcárias.

O uso intensivo de modernas tecnologias na produção de melão no semiárido vem aumentando, a cada ano, em consequência das exigências do mercado interno e externo por produtos de qualidade. Esse crescimento tecnológico da agricultura, sobretudo a partir da segunda metade do século XX, incorporou um conjunto de tecnologias avançadas, as quais aumentaram a produção e alteraram as relações sociais no campo (Schons \& Miorin, 2007). Porém, a incorporação dessas novas tecnologias ao meio rural brasileiro ocorreu, muitas vezes, de forma inadequada à realidade do meio, com consequências altamente negativas sobre o ambiente natural (Rocha et al., 2009). Para Rocha et al. (2009), com a modernização da agricultura e a expansão do agronegócio no mundo, o uso desregrado do solo gerou sérios problemas de erosão, reduzindo a espessura e a fertilidade dos solos e diminuindo drasticamente a produtividade em algumas áreas. Com o manejo inadequado e intensivo do solo, podese ocasionar um estado de degradação que, caso seja reversível, requer muito tempo e recurso para a sua recuperação (Mendes et al., 2002). Assim, faz-se necessária a preservação do solo, manejando de forma sustentável, mantendo a qualidade e continuidade da produção.
A manutenção da produtividade dos ecossistemas agrícolas e florestais depende, em grande parte, do processo de transformação da matéria orgânica e, por conseguinte, da biomassa microbiana (Gama-Rodrigues et al., 2008). Segundo Trannin et al. (2007), a atividade microbiana do solo é responsável pela decomposição dos compostos orgânicos, pela ciclagem de nutrientes e pelo fluxo de energia do solo. A biomassa microbiana e sua atividade têm sido apontadas como as características mais sensíveis às alterações na qualidade do solo, causadas por mudanças de uso e por práticas de manejo. Estudos elaborados por Venzke Filho et al. (2008) mostraram que a quantidade e a composição da biomassa microbiana podem ser influenciadas por diversos fatores, entre eles o sistema de cultivo, a rotação de cultura e a textura do solo.

A quantidade de matéria orgânica (MO) está relacionada com os aportes de resíduos orgânicos e à formação de biomassa vegetal, a qual, por sua vez, depende do nível de fertilidade do solo. Para Salcedo \& Sampaio (2008), a MO deve ser conservada e manejada de forma equilibrada de forma que permita o desenvolvimento de uma agricultura sustentável, pelo uso de métodos adequados de manejo de solo, compreendendo sistemas de preparo, rotação de culturas, adubação, estabelecimento de sistemas integrados lavoura-pastagem, dentre outros. Suas funções no solo, especialmente sob condições tropicais e subtropicais, conferem estabilidade aos agregados e à estrutura do solo, infiltração e retenção de água, resistência à erosão, capacidade de troca de cátions (CTC), disponibilidade de nutrientes, aeração e atividade da biomassa microbiana (Rossato et al., 2004).

O objetivo deste trabalho foi avaliar as alterações microbiológicas do solo, assim como o teor de matéria orgânica nas profundidades $0-0,10,0,10-0,20,0,20-0,30 \mathrm{e}$ 0,30-0,40 m, sob o cultivo do melão, durante três, cinco e dez anos, em Cambissolo Háplico eutrófico, a fim de compará-los com os da mata natural de predomínio local, a Caatinga. 


\section{MATERIAL E MÉTODOS}

As coletas foram realizadas na fazenda Melão Doçura, localizada no município de Quixeré, Ceará. O município encontra-se sob as coordenadas geográficas latitude $-05^{\circ}$ 04'27' S, longitude -3759'19' W e altitude 25 m. Apresenta clima tropical quente semiárido, com temperatura média variando entre 26 e $28^{\circ} \mathrm{C}$ e precipitação de $857,7 \mathrm{~mm}$ (FUNCEME, 2009).

As amostras de solo foram retiradas, nas profundidades de 0-0,10, 0,10-0,20, 0,20-0,30 e 0,30-0,40 m, de um Cambissolo Háplico eutrófico (Embrapa, 2006), em áreas sob mata natural (Caatinga) e de áreas sob cultivo do melão,durante três, cinco e dez anos. Na área sob cultivo, o solo foi preparado com o uso de grade aradora, subsolador, grade niveladora, adubadora e, entre outras operações, como pulverizações, cultivo mecânico e uso de carroções para transporte dos frutos, seguindo-se o mesmo preparo do solo para todos os anos de cultivo. No período de entressafra, utilizou-se milho (Zea mays L.), sorgo (Sorghum bicolor L.) e mucuna (Mucuna pruriens L.) em rotação de culturas e para incorporação de massa verde, respecivamente. Na Tabela 1, estão apresentadas as característica físicas e químicas do Cambissolo Háplico eutrófico, coletado, na profundidade de 0 a $0,10 \mathrm{~m}$.

As amostras foram secas ao ar, posteriormente destorroadas e passadas por peneira de malha $2 \mathrm{~mm}$. A quantificação de carbono da biomassa microbiana foi realizada em extrato de amostras de solos fumigadas e não fumigadas, após período de 24 horas de ativação de microorganismos, com solos saturados em recipiente fechado. A fumigação foi feita em aparelho de micro-ondas, por 4,5 minutos; e, em seguida, fez-se a extração. Utilizou-se como extrator sulfato de potássio $0,5 \mathrm{M}$, a pH 6,5, na relação 1:2,5 (solo:extrator), tanto para amostras fumigadas quanto para as não fumigadas, usando-se método proposto por Vance et al. (1987) e descrito por De-Polli \& Guerra (1999). Foram feitos a extração e fracionamento da matéria orgânica do solo (Duarte, 1994) e a quantificação do carbono microbiano, utilizando-se o método de colorimetria, descrito por Bartlett \& Ross (1988). Foi feita a leitura da absorbância da curva padrão e das amostras, em espectrofotômetro, utilizando- se um comprimento de ondas de $495 \mathrm{~nm}$. O cálculo da concentração de carbono foi feito, utilizando-se a equação de reta obtida a partir da curva padrão:

$$
\mathrm{C}(\mathrm{mg} / \mathrm{L})=\left(\frac{A b s-a}{b}\right) \frac{V}{P} f, \text { em que: }
$$

$C=$ concentração de Carbono; $A b s=$ absorbância da amostra, $a=$ intercepto da reta encontrada na curva, $b=$ coeficiente de declividade da reta, $V=$ volume usado na extração, $P=$ massa seca do solo e $f=$ fator de diluição da curva. O quociente microbiano ( $q \mathrm{MIC}$ ) foi calculado pela razão entre o carbono da biomassa microbiana e o carbono orgânico do solo, afim de refletir os aportes de carbono e a conversão de substratos orgânicos para o carbono da biomassa microbiana (Sparling, 1997). Para Wardle et al. (1994), este quociente pode avaliar a qualidade da matéria orgânica, enquanto, para Ferreira (2008), trata-se de um indicador da sua dinâmica, expressando a eficiência da biomassa em utilizar $\mathrm{C}$ e $\mathrm{N}$.

O delineamento experimental utilizado foi o inteiramente casualizado (DIC), disposto em esquema fatorial $4 \times 4$, com três repetições. O Fator I foi a causa de variação na profundidade ( 0 a $0,10 \mathrm{~m}, 0,10$ a $0,20 \mathrm{~m}, 0,20$ a $0,30 \mathrm{~m} \mathrm{e} 0,30$ a 0,40 m) e, o Fator II, os anos de cultivo (0, 3, 5 e 10 anos). Os dados foram submetidos à análise de variância, sendo as médias comparadas pelo teste Scott-Knott, a 5\% de probabilidade. Todas as análises foram realizadas com o auxílio do software SISVAR 4.3 (Ferreira, 2003).

\section{RESULTADOS E DISCUSSÃO}

Na interação profundidade/tempo, verificou-se diferença significativa de 5\%, pela análise de variância, da variação da matéria orgânica (MO). Porém, para a variação do carbono da biomassa microbiana (CBM) e do quociente microbiano ( $q \mathrm{MIC}$ ), as diferenças foram significativas, a $1 \%$ somente para profundidade e tempo respectivamente, não havendo diferença significativa na interação dos fatores avaliados pelo teste de Scott-knott.

Observou-se, na Figura 1, maior destaque para a camada superficial na mata natural, apresentando valores mais altos, devidos à presença de cobertura morta, nessa área, e ao decréscimo desta, à medida que os pontos de coleta

Tabela 1. Características físicas e químicas do Cambissolo Háplico eutrófico coletado em Caatinga na profundidade de 0 a $0,10 \mathrm{~m}$

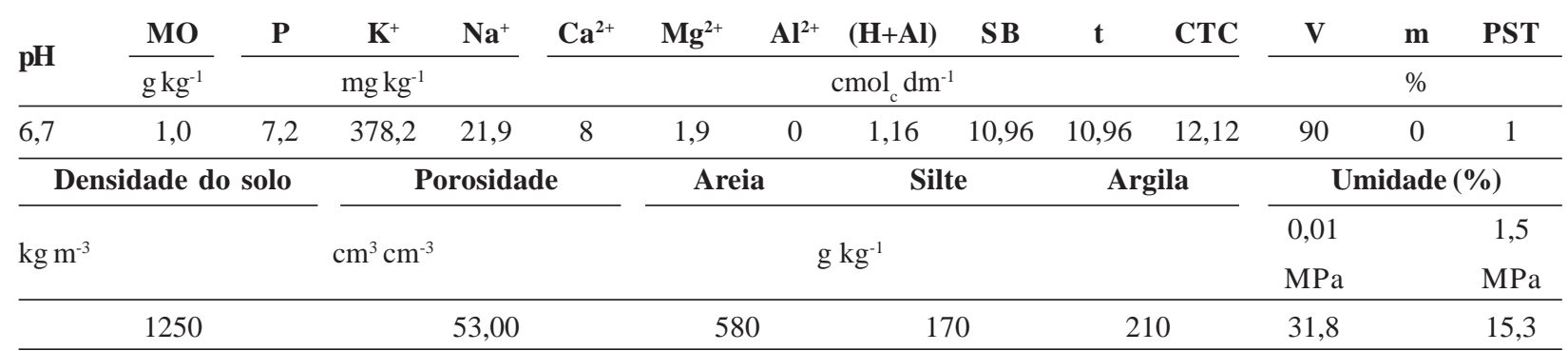

*MO = matérias orgânica $\left(\mathrm{g} \mathrm{kg}^{-1}\right)$; PST = Percentagem de sódio trocável; e CTC = capacidade de troca de cátions. 
tornaram-se mais profundos. A concentração de MO nas profundidades de 0 a $0,10 \mathrm{~m}$ e 0,10 a $0,20 \mathrm{~m}$, encontraram-se elevadas, durante todos os anos de cultivo do melão. As maiores concentrações são detectadas na camada superficial do solo, em razão do aporte de resíduos nesta camada.

No cultivo do melão durante dez anos, foi possível observar, nas profundidades 0,20 a 0,30 m e 0,30 a 0,40 m, acréscimo significativo de $\mathrm{MO}$, quando comparada com a da Caatinga, evidenciando-se acúmulo de MO nessas profundidades. Em vista da carência de estudos que correlacionem o tempo de cultivo com o sistema de manejo, esses fatos podem ser explicados pelo uso adequado do manejo, acúmulo de resíduos vegetais durante os anos de pousio, o tempo de cultivo e rotação de culturas, como milho, sorgo e mucuna, como, também, a incorporação desta ao solo, proporcionando o que se chama de acréscimo significativo de MO em profundidade, visualizável a partir dos cinco anos de cultivo (Figura 1).

Addiscot (1992) assinala, em seu estudo que, na natureza, o solo comporta-se como um sistema aberto, trocando matéria orgânica e energia com o meio, e o sistema atinge um estado estável quando as taxas de adição e de perdas equivalem-se. Mielniczuk (2008) relata que o declínio da matéria orgânica ao longo do tempo indica erro no sistema de manejo, como baixa fertilidade, baixa produção de resíduo, excesso de revolvimento, erosão acelerada, dentre outros problemas.

$\mathrm{O}$ carbono da biomassa microbiana foi significativo em profundidade, a $1 \%$ de probabilidade, pelo teste de Scott-Knott, e apresentou efeito não significativo na interação profundidade/tempo.

O solo Caatinga revelou carbono microbiano mais elevado que o das demais áreas analisadas, podendo esse fato ser atribuído à maior concentração de MO na camada superior do solo (Figura 2). O valor mais elevado de carbono da biomassa microbiana no solo, sob a mata natural, deve-se ao aporte contínuo e diversificado de matéria orgânica incorporada ao solo, principalmente via deposição pelas plantas. Segundo Matsuoka et al. (2003), a di-

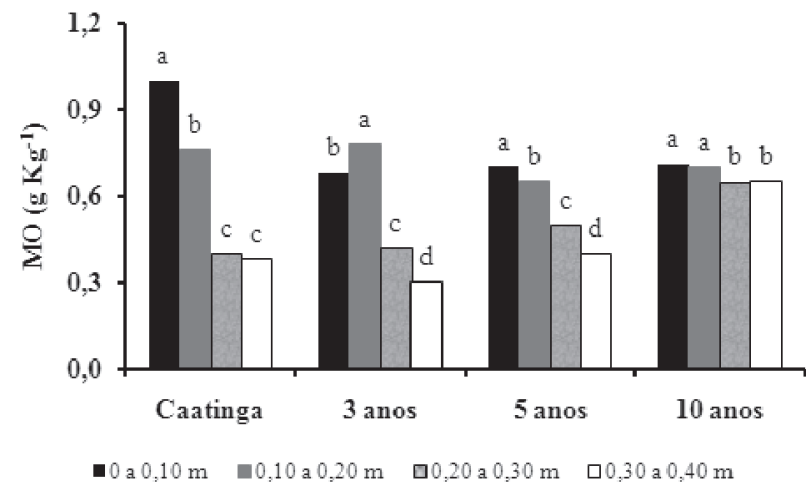

Figura 1. Valores de Matéria Orgânica na variável profundidade/ tempo. versidade florística das áreas nativas e a presença da vegetação durante todo ano influenciam a quantidade e qualidade da serrapilheira, fato este que pode ter contribuído para um maior acúmulo de carbono pela biomassa microbiana, e, consequentemente, para melhores condições de desenvolvimento da microbiota (Figura 2).

As profundidades mais favoráveis ao crescimento do CBM foram observadas na camada superficial, de 0 a $0,10 \mathrm{~m}$ e 0,10 a $0,20 \mathrm{~m}$, durante todos os anos de cultivo; entretanto, na profundidade de $30-40 \mathrm{~cm}$, nos períodos de cultivo de cinco e dez anos, os resultados observados estiveram próximos aos da Caatinga. Esses fatos poderiam ser explicados pela grande demanda de MO nessas profundidades, como pelo tipo de manejo utilizado, já que a biomassa microbiana é extremamente sensível ao manejo dado ao solo.

Segundo Powlson et al. (1987), a biomassa microbiana é sensível às alterações nas formas de carbono orgânico do solo, em função das mudanças no manejo ou uso do solo. Para o mesmo autor, após as alterações serem introduzidas, a biomassa microbiana sofrerá flutuações, até atingir um novo equilíbrio. Estudos elaborados por Perez et al. (2004); Conceição et al. (2005) e Ferreira et al. (2007) mostraram que o teor de carbono da biomassa microbiana mantiveram-se significativamente maiores na camada de 0 a $0,10 \mathrm{~m}$ de profundidade, independentemente das características das áreas estudadas. Reduções do carbono da biomassa microbiana foram observadas em estudos realizados por Matsuoka et al. (2003) em solos do Cerrado, nos quais verificaram-se diminuições nos teores em profundidades maiores, estando, na camada superficial, os seus maiores teores. Teores mais elevados de CBM implicam maior imobilização temporária de nutrientes e, consequentemente, menores perdas de nutrientes no sistema solo-planta (Mercante, 2004).

O quociente microbiano ( $q \mathrm{MIC}$ ) foi significativo para o fator tempo, a $1 \%$ de probabilidade pelo teste de ScottKnott, e apresentou efeito não significativo na interação profundidade/tempo.

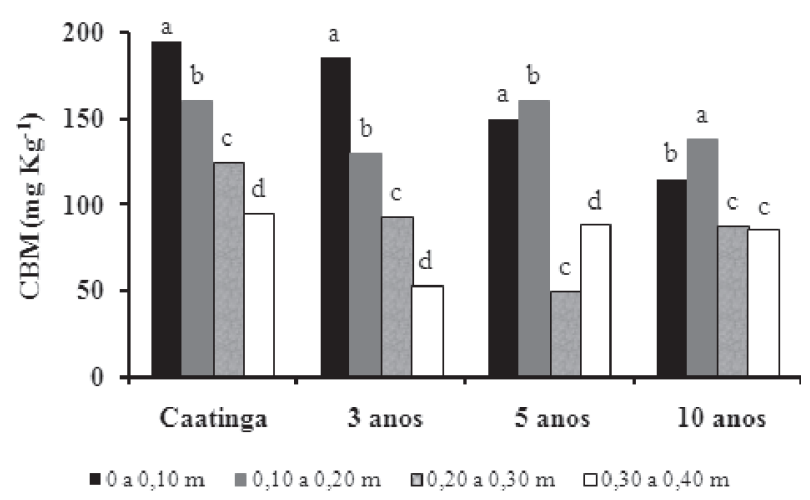

Figura 2. Valores do Carbono da biomassa microbiana na variável profundidade/tempo. 
Observa-se, na Figura 3, o aumento significativo do $q \mathrm{MIC}$, sob a Caatinga, nas profundidades 20-30 e 30-40 $\mathrm{cm}$. De acordo com Anderson \& Domsch (1989), um maior $q \mathrm{MIC}$ representa maior ciclagem de nutrientes e, portanto, menor acúmulo de carbono. Além disso, os maiores valores da proporção indicam a maior conversão do COT em CBM (Marchiori Júnior \& Mello, 2000) (Figura 3).

As mudanças na relação $q \mathrm{MIC}$ refletem o padrão de entrada de MO nesses solos, a eficiência da conversão do $\mathrm{C}$ microbiano, as perdas do $\mathrm{C}$ do solo e a estabilização do C orgânico pela fração mineral do solo (Sparkling, 1992). O nível da relação do $q \mathrm{MIC}$ pode indicar se o carbono está em equilíbrio, se está acumulando ou diminuindo (Insam, 1990).

Na Figura 3, observa-se, também, que, durante os anos de cultivo, houve decréscimo do $q \mathrm{MIC}$, com o tempo, e oscilações em profundidade; porém, dez anos de cultivo do melão apresentou menor $q \mathrm{MIC}$. Trabalhos têm demonstrado que os quocientes microbianos ( $q \mathrm{Mic})$ e metabóli$\cos \left(q \mathrm{CO}_{2}\right)$ são sensíveis aos efeitos ambientais e antropogênicos sobre a comunidade microbiana do solo, podendo-se constituir indicadores das perturbações dos ecossistemas (Andréa et al., 2004; Harris, 2003).

Wardle et al (1994) afirma que os baixos teores de $q \mathrm{MIC}$ podem ocorrer por estar a microbiota sob algum fator de estresse, ou, como propõem Gama-Rodrigues et al. (2008), por causa da baixa qualidade nutricional da matéria orgânica, fazendo com o que a biomassa microbiana torne-se incapaz de utilizar totalmente o carbono orgânico total.

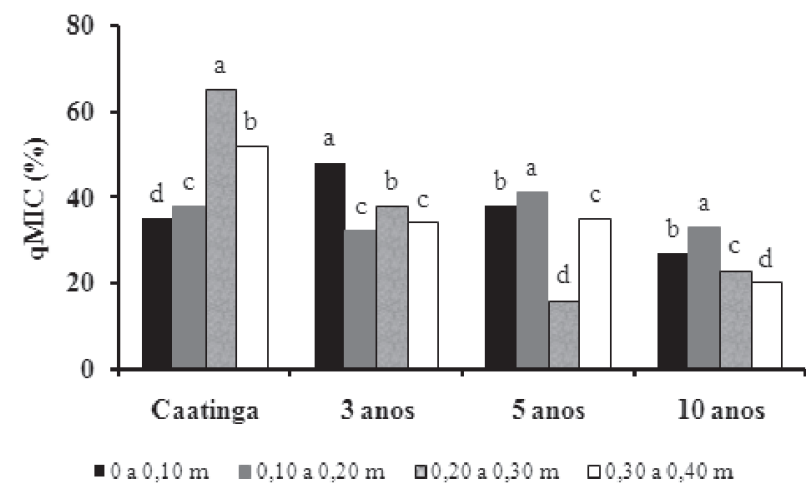

Figura 3. Valores de quociente microbiano na variável profundidade/tempo.

\section{CONCLUSÕES}

Houve aumento significativo da matéria orgânica, em profundidade, a partir dos cinco anos de cultivo do melão.

O carbono da biomassa microbiana aumentou nas camadas superficiais do solo, independentemente dos anos de cultivo, por causa da maior disponibilidade da matéria orgânica, água e nutrientes.
O quociente microbiano diminuiu com o tempo, apresentando menor concentração no cultivo de dez anos do melão, discriminando as possíveis alterações decorrentes do uso agrícola do solo.

\section{REFERÊNCIAS BIBLIOGRÁFICAS}

Addiscot TM (1992) Entropy and sustainability. European Journal of Soil Science, 46:161-168.

Anderson TH \& Domsch KH (1989) Rations of microbial biomass carbon to total organic in arable soils. Soil Biology \& Biochemistry, 21:474-479.

Andréa AFD, Silva MLN, Curi N \& Guilherme LRG (2004) Estoques de carbono e nitrogênio e formas de nitrogênio mineral em um solo submetido a diferentes sistemas de manejo. Pesquisa Agropecuária Brasileira, 39:179-186.

Bartlett RJ \& Ross D S (1988) Colorimetric determination of oxidizable carbon in acid soil solution. Soil Science Society of America Journal, 52:1191-1192.

Conceição PC, Amado TJC, Mielniczuk J \& Spagnollo E (2005) Qualidade do solo em sistemas de manejo avaliada pela dinâmica da matéria orgânica e atributos relacionados. Revista Brasileira de Ciência do Solo, 29:777-788.

De-Polli H \& Guerra JGM (1999) C, N e P na biomassa microbiana do solo. In: Silva LS, Canellas LP \& Camargo FAO (Eds.) Fundamentos da matéria orgânica do solo: ecossistemas tropicais e subtropicais. Porto Alegre, Gênesis. p.389-411.

Duarte PA (1994) Calagem e sistema de rotação de culturas: efeitos nas características e propriedades da matéria orgânica do solo. Dissertação de mestrado. Universidade Estadual Paulista "Júlio de Mesquita Filho", Jaboticabal. 165p.

Embrapa (2006) Sistema brasileiro de classificação de solos. 2.ed. Rio de Janeiro: Centro Nacional de Pesquisa de Solos. 306p.

Ferreira EAB, Resck DVS, Gomes AC \& Ramos MLG (2007) Dinâmica do carbono da biomassa microbiana em cinco diferentes épocas do ano em diferentes sistemas de manejo do solo no cerrado. Revista Brasileira de Ciência do Solo, 31:1625-1635.

Ferreira GM (2008) Atividade microbiana e agregação de um Latossolo Vermelho Distroférrico em Campinas, SP, sob usos e manejos distintos. Dissertação de Mestrado. Instituto Agronômico de Campinas, Campinas. 70p.

Ferreira DF (2003) Programa Sisvar. Versão 4.6. Universidade Federal de Lavras, Lavras.

Funceme (2009) Governo do Estado do Ceará. Disponível em: <http://www.ipece.ce.gov.br/publicacoes/perfil_basico/pbm2009/ Quixere_Br_office.pdf>. Acessado em: 10 de junho 2012.

Gama-Rodrigues EF, Gama-Rodrigues AC, Paulino GM \& Franco AA (2008) Atributos químicos e microbianos de solos sob diferentes coberturas vegetais no norte do estado do Rio de Janeiro. Revista Brasileira de Ciência do Solo, 32:1521-1530.

Harris JA (2003) Measurements of the soil microbial community for estimating the success of restoration. European Journal Soil Science, 54:801-808.

Insam H (1990) Are the soil microbial biomass and basal respiration governed by the climatic regime? Soil Biology and Biochemistry, 22:525-532.

Mielniczuk JEM (2008) Matéria Orgânica e a sustentabilidade de sistemas agrícolas. In: Santos GA, Silva LS da, Canellas LP \& Camargo FAO (Eds.) Fundamentos da Matéria Orgânica do Solo: ecossistemas tropicais \& subtropicais. Porto Alegre, Gênesis. p.1-5.

Rev. Ceres, Viçosa, v. 59, n.4, p. 565-570, jul/ago, 2012 
Matsuoka M, Mendes IC \& Loureiro MF (2003) Biomassa microbiana e atividade enzimática em solos sob vegetação nativa e sistemas agrícolas anuais e perenes na região de Primavera do Leste (MT). Revista Brasileira de Ciência do Solo, 27:425433.

Marchiori Júnior M \& Melo WJ (2000) Alterações na matéria orgânica e na biomassa microbiana em solo de mata natural submetido a diferentes manejos. Pesquisa Agropecuária Brasileira, 35:1177-1182.

Mercante FM (2004) Parâmetros microbiológicos como indicadores de qualidade do solo sob sistemas integrados de produção agropecuária. Dourados, Embrapa Agropecuária Oeste. 27p. (Boletim de Pesquisa e Desenvolvimento, 20).

Mendes IC, Reis Júnior F \& Perreira Neto JV (2002) Uso de indicadores biológicos e bioquímicos para avaliar a qualidade de solos de cerrado sob plantio direto e convencional. In: $26^{\mathrm{a}}$ Reunião Brasileira de Fertilidade do Solo e Nutrição de Plantas, $9^{a}$ Reunião Brasileira sobre Micorrizas, $7^{\circ}$ Simpósio Brasileiro de Microbiologia do Solo, $4^{\text {a }}$ Reunião Brasileira de Biologia do Solo, 2002. FERTBIO2002: Agricultura: bases ecológicas para o desenvolvimento social e econômico sustentado, Rio de Janeiro. Anais, SBM. p.54.

Perez KSS, Ramos MLG \& McManus C (2004) Carbono da biomassa microbiana em solo cultivado com soja sob diferentes sistemas de manejo nos Cerrados. Pesquisa Agropecuária Brasileira, 39:567-573.

Powlson DS, Brookes PC \& Christensen BT (1987) Measurement of soil microbial biomass provides an early indication of changes in total soil organic matter due to straw incorporation. Soil Biology \& Biochemistry, 19:159-164.

Rocha MC, Gonçalves LSA, Corrêa FM, Lima Silva RRS, Abboud ACS \& Carmo MGF (2009) Descritores quantitativos na determinação da divergência genética entre acessos de tomateiro do grupo cereja. Ciência Rural, 39:664-670.
Rossato L, Alvalá RCS \& Tomasella J (2004) Variação espaçotemporal da umidade do solo no Brasil: análise das condições médias para o período de 1971-1990. Revista Brasileira de Meteorologia, 19:113-122.

Salcedo IH \& Sampaio EVSB (2008) Matéria orgânica do solo no bioma caatinga. In: Santos GA, Silva LS, Canellas LP \& Camargo FAO (eds.) Fundamentos da matéria orgânica do solo: Ecossistemas tropicais e subtropicais. 2.ed. Porto Alegre, Metrópole, p.419-441.

Schons MI \& Miorin VMF (2007) Uso da terra no espaço rural do município de Campina das Missões, RS. Revista Brasileira de Agroecologia, 2:1182-1185.

Sparkling GP (1992) Ratio of microbial biomass carbon to soil organic carbon as a sensitive indicator of changes in soil organic matter. Australian Journal of Soil Research, 39:195-207.

Trannin ICB, Siqueira JO \& Moreira FMS (2007) Características biológicas do solo indicadoras de qualidade após dois anos de aplicação de biossólido industrial e cultivo de milho. Revista Brasileira de Ciência do Solo, 31:1173-1184.

Vance ED, Brooks PC \& Jenkinson DS (1987) An extraction method for measuring soil microbial biomass C. Soil Biology and Biochemistry, 19:703-707.

Venzke Filho SP, Feigl BJ, Piccolo MC, Siqueira Neto M \& Cerri CC (2008) Biomassa microbiana do solo em sistema de plantio direto na região de Campos Gerais - Tibagi, PR. Revista Brasileira de Ciência do Solo, 32:599-610.

Wardle DA, Nicholson KS, Ahmed M \& Rahman A (1994) Interference effects of the invasive plant Carduus nutans L. against the nitrogen fixation ability of Trifolium repens L. Plant and Soil, 163:287-297. 